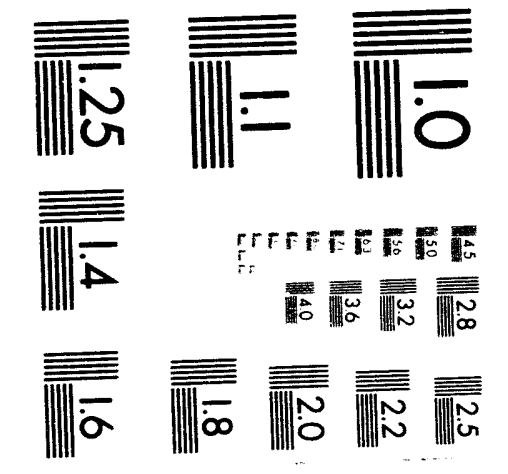



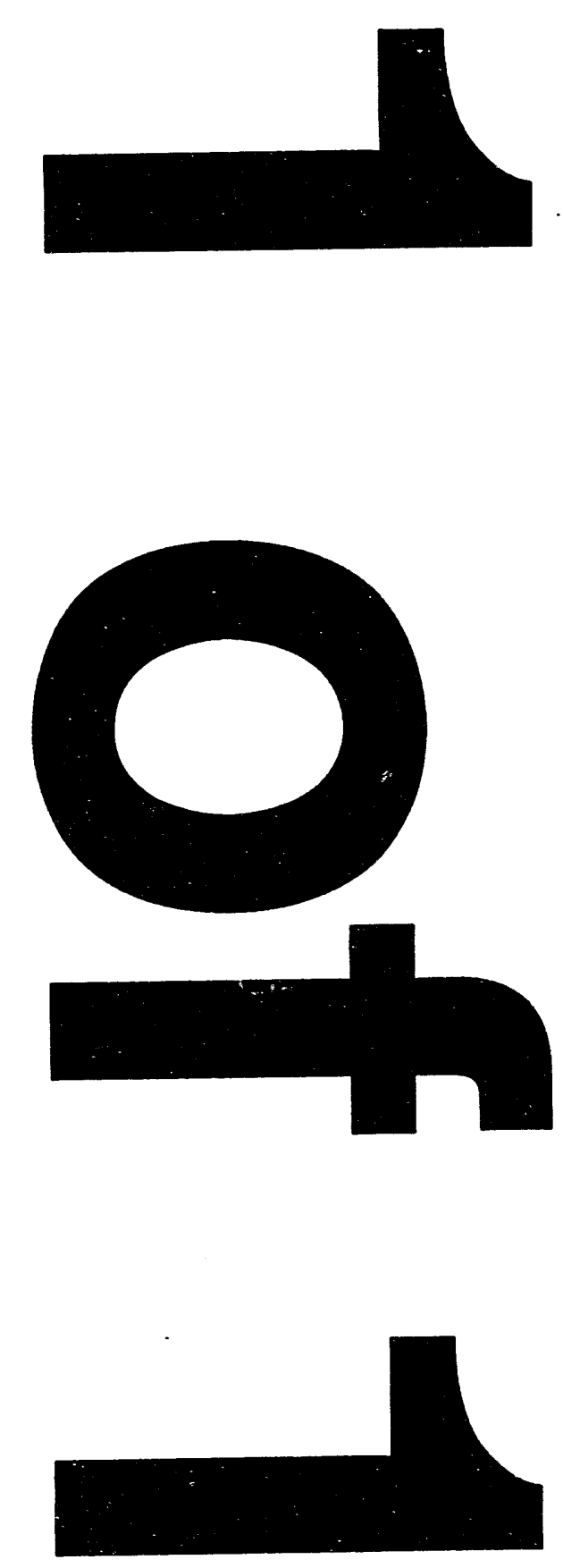


\title{
Analytical Solutions of Minimum Ionization Particle Induced Current Shapes of Silicon Detectors and Simulation of Charge Collection Properties*
}

\author{
V. Eremin \\ A. F. Ioffe Physico-Technical Institute of Academy of Sciences of Russia, St. Petersburg, \\ Russia \\ W. Chen, Zheng Li \\ Brookhaven National Laboratory, Upton, NY 11973 USA
}

November 1993

DISCLAIMER

\begin{abstract}
This report was prepared as an account of work sponsored by an agency of the United States Government. Neither the United States Government nor any agency thereof, nor any of their employees, makes any warranty, express or implied, or assumes any legal liability or responsibility for the accuracy, completeness, or usefulness of any information, apparatus, product, or process disclosed, or represents that its use would not infringe privately owned rights. Reference herein to any specific commercial product, process, or service by trade name, trademark, manufacturer, or otherwise does not necessarily constitute or imply its endorsement, recommendation, or favoring by the United States Government or any agency thereof. The views and opinions of authors expressed herein do not necessarily state or reflect those of the United States Government or any agency thereof.
\end{abstract}

*This research was supported by the U.S. Department of Energy: Contract No. DE-AC02-76CH00016. 


\title{
Analytical Solutions of Minimum Ionization Particle induced Current Shapes of Silicon Detectors and Simulation of Charge Collection Properties
}

\author{
V. Eremin \\ A. F. Ioffe Physico-Technical Institute of Academy of Sciences of Russia, St. Petersburg, Russia \\ W. Chen and $\mathrm{Z}$. $\mathrm{Li}$ \\ Brookhaven National Laboratory Upton, NY 11973-5000, USA
}

\section{Abstract}

A new analytical, one dimensional method to obtain the induced current shapes and simulation of charge shapes for $\mathrm{p}^{+}-\mathrm{n}-\mathrm{n}^{+}$silicon detectors in the case of minimum ionization particle has been developed here. Exiact solutions have been found for both electron and hole current shapes. Simulations of induced charge shapes of detectors have also been given. The results of this work are consistent with the earlier work where a semi-analytical method had been used.

\section{INTRODUCTION}

Charge collection propertics of silicon detector under high fluence of neutron radiation in the proposed large colliders, such as the SSC and LHC, is an important effect of radiation damage in detectors. There have been some modeling and simulation works done semi-inalytically in the past about induced current shipes for alpha particle as well as minimum ionization particle (MIP), and trapping effects[1]. In this work we report a new antlytical, one dimensional method to obtain the induced current shapes and simulation of charge shapes for $\mathrm{p}^{+}-11-11^{+}$silicon detector in the case of MIP. Here the diffusion and detrapping effects are not taken into account. The trapping effect will be considered in the paper.

\section{ANALYTICAL SOLUTIONS}

The electron and hole densities are denoted as $n(t)$ and $p(t)$ respectively, with initial values of $n(0)$ and $p(0)$. In order to have field distribution in the detector not being disturbed by $n(t)$ and $p(t)$, the signal level must be low, i.e. $n(t) \ll N_{e f f} p(t) \ll N_{e f f}$. In the calculations. the bials used is a constant, the space charge density is defined as $\rho=e N_{(j)}$; and detector thickness is $d$.

\section{A. Calculation of electron pulse shape}

The field distribution in the detector is shown in Fig. I where the shaded area is the layer of moving electrons. The boundaries of the charge layers $\xi(t)$ are assumed to move with velocity $\mu_{n} E(x)$ for electrons and $\mu_{p} E(x)$ for holes. The track length for the boundary layer of charges is defined as $l(t)$ which is related to $\xi(t)$ as following:

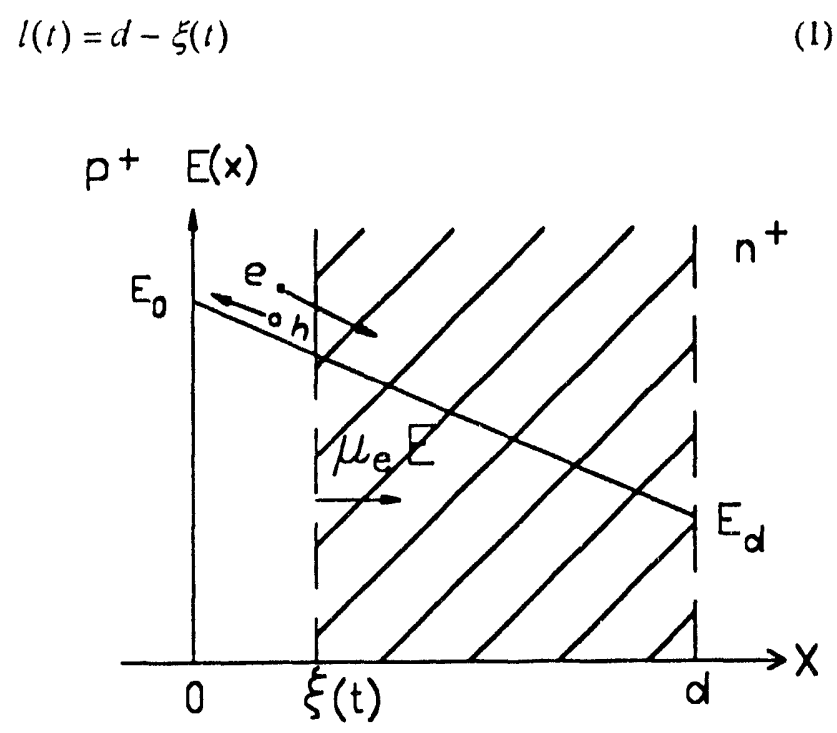

Fig. 1. Field distribution in detector. Shaded area is the layer of moving electrons

The field distribution is:

$E(x)=E_{0}-\frac{\rho}{\varepsilon \varepsilon_{0}} x$

where $\rho=e N_{e j f}$ and $E_{O}$ is defined as:

$E_{0}=\frac{V}{d}+\frac{d p}{2 \varepsilon \varepsilon_{0}}$

The current density of electrons $i_{e}(x, t)$ at $x \geq \xi(t)$ is

$i_{v}(x, t)=\mu_{\mathrm{e}} \operatorname{en}(x, t) E(x)+\varepsilon \varepsilon_{0} \frac{\partial E(x, t)}{\partial t}$ 
$(E(x, t)$ is taken into account only in the calse of displacement current). The current $i_{e}(x, t)$ is a constant at each cross-section of the detector according the Kizhyoft law. Integrating the current of equation (t) along the detector's thickness we have

$i_{e}=\frac{1}{d} \mu_{e} e \int_{\xi(t)}^{d} n(x, t) E(x) d x$

For each separate point (layer), we can write the following formula for $n(x, t)$ :

$\frac{d n(x, t)}{d t}=\frac{\partial n}{\partial t}+\mu E \frac{\partial n}{\partial x}$

From continuation law we have:

$\frac{\partial i}{\partial x}=-e \frac{\partial n}{\partial t}$

Combining equation (4), (7) and using Poisson equation

$\frac{\partial E}{\partial x}=-\frac{\rho}{\varepsilon \varepsilon_{0}}$, we get

$\frac{\partial n}{\partial t}+\mu E \frac{\partial n}{\partial x}=\frac{\ln \rho}{\varepsilon \varepsilon_{0}}$

Substitute (8) into (6) we have

$$
\frac{d n}{d t}=\frac{\mu m \rho}{\varepsilon \varepsilon_{0}}
$$

Solution of (9) is

$n(t)=n_{0} e^{\frac{\mu \rho}{\varepsilon \varepsilon_{0}}}$

It means that concentration in cach layer of moving trick increases in time with rate $\frac{\mu \rho}{\varepsilon \varepsilon_{0}}$, which does not depend on coordinate $x$. So the homogencous concentration in the track is conserved with time. From (5) ind (l(1) we get $i_{v}(t)=\frac{1}{d} \mu_{e} e n_{0} e^{\frac{\mu_{\rho} \rho}{\varepsilon \varepsilon_{1}}}\left[E_{0}(d-\xi(t))-\frac{\rho}{2 \varepsilon \varepsilon_{0}}\left(d^{2}-\xi^{2}(t)\right)\right]$

where $\xi(t)$ is the solution of the following equation:

$\frac{\partial \xi}{\partial t}=\mu E(\xi)=\mu E_{0}\left(1-\frac{\xi \rho}{\varepsilon \varepsilon_{0} E_{0}}\right)$

and is found:

$\xi(t)=E_{0} \frac{\varepsilon \varepsilon_{0}}{\rho}\left(1-e^{-\frac{\mu \rho}{\varepsilon \varepsilon_{0}} t}\right)$

We note here that $t$ should be limited by the value of collection time $i_{c}$ According to equation (13) we know that at $t=t_{c}^{e}, \xi=d$, we get

$t_{c}^{e}=\frac{\varepsilon \varepsilon_{0}}{\mu_{\iota} \rho} \ln \frac{\frac{2 V \varepsilon \varepsilon_{0}}{\rho l^{2}}+1}{\frac{2 V \varepsilon \varepsilon_{0}}{\rho d^{2}}-1}$

The current shape for clectrons calculated from equation (11) and equation (13) is shown in Fig. 2. The values used for electron and hole mobilities in this paper are $1+50$ $\mathrm{cm}^{2} / \mathrm{v} / \mathrm{s}$ and $+50 \mathrm{~cm}^{2} / \mathrm{v} / \mathrm{s}$, respectively.

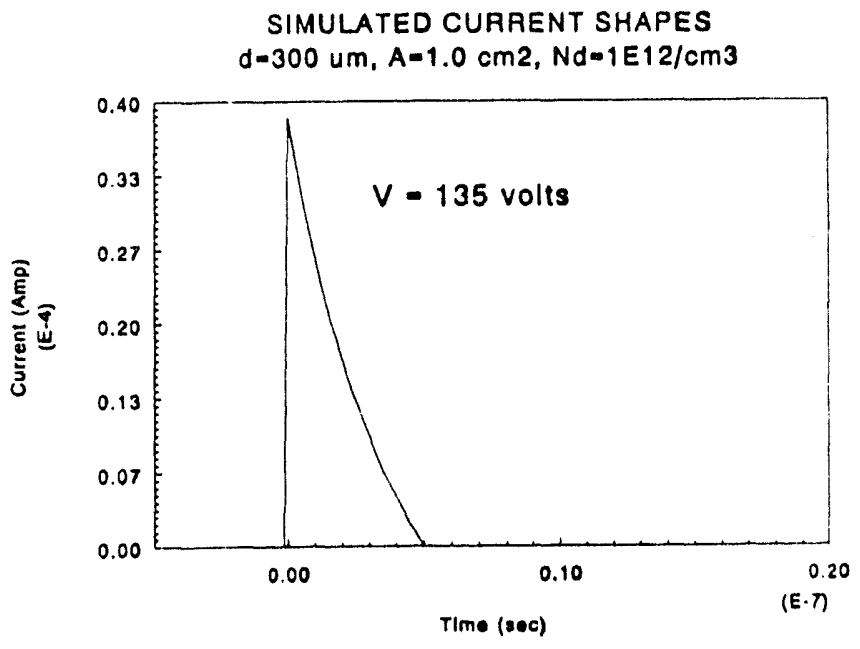

Fig. 2. The electron current shape simulation result for MIP 


\section{B. Calculation of hole pulse shape}

Similarly, we can calculate the hole current shape using the same method described above. The licld distribution in the detector is shown in Fig. 3. Again, the hole current can be written as:

$i_{p}(t)=\frac{1}{d} \mu_{p} e^{\xi(t)} \int_{0} p(x, 1) E(x) d x$ $\xi_{p}(t)=d e^{\frac{\mu \mu_{p}}{\varepsilon \varepsilon_{0}}}+\frac{E_{0} \varepsilon \varepsilon_{0}}{\rho}\left(1-e^{\frac{\mu_{p} \rho}{\varepsilon \varepsilon_{0}}}\right)$

The collection time of holes $t_{c}^{p}$ can be found from equation (18) using $\xi_{p}\left(t_{c}^{p}\right)=0$ :

$t_{c}^{p}=\frac{\varepsilon \varepsilon_{0}}{\mu_{\rho} \rho} \ln \frac{\frac{2 V \varepsilon \varepsilon_{0}}{\rho d^{2}}+1}{\frac{2 V \varepsilon \varepsilon_{0}}{\rho d^{2}}-1}$

We note that the expression for collection time for holes $t_{c}^{p}$ is similar to that for electrons (equation (14)), with the only difference in the carrier mobilities: $\mu_{p}$ for holes and $\mu_{e}$ for electrons.

The hole current shape calculated from equation (17) to equition (19) is shown in Fig. 4.

Fig. 3. Field distribution in detector. Shaded area is the layer of moving holes.

Similar to the solution of equation (6) to equation (y), we obtain the following cxpression for $p(x, t)$ :

$p(t)=p_{j} e^{-\frac{\mu_{j} \rho_{1}}{\varepsilon \varepsilon_{0}}}$

So for holes, the concentration decreases with time becilluse holes move in the direction of increasing of ficld. In this case the front layer of holes has greater velocity than the back layer. From (15) and (16) we get the hole current shape:

$i_{p}(t)=\frac{\mu_{p} e p_{0}}{d} e^{-\frac{\mu_{p} p}{\varepsilon \varepsilon_{0}} t}\left[E_{0} \xi_{p}(t)-\frac{\rho}{2 \varepsilon \varepsilon_{0}} \xi^{2}(l)\right]$

Where $\xi_{p}(t)$ is:

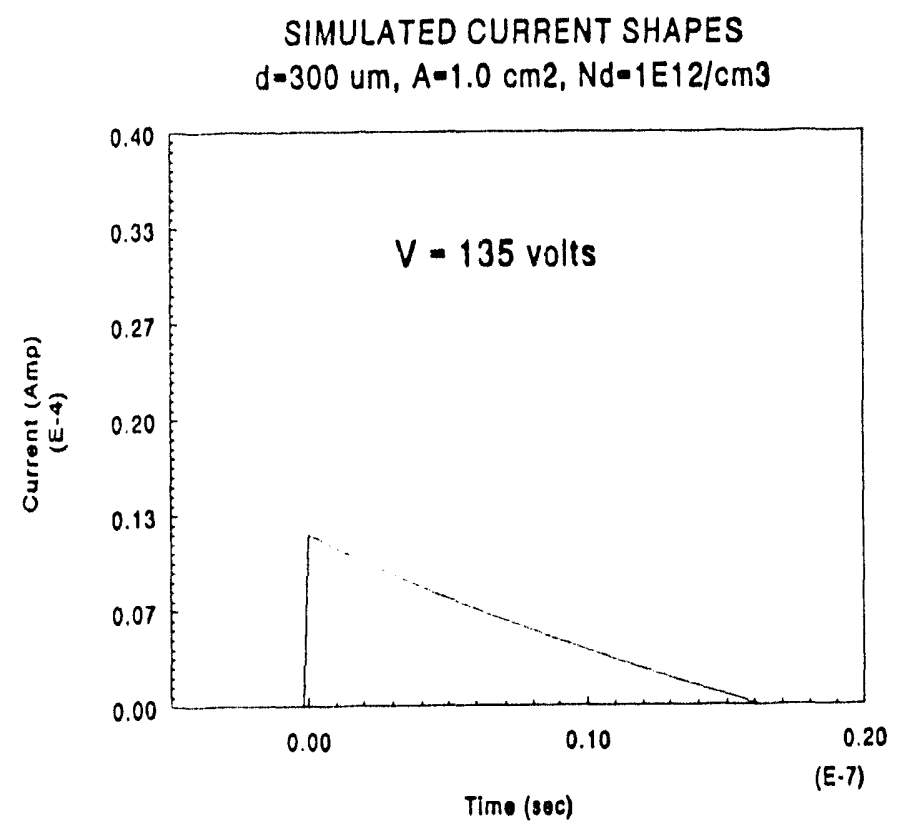

Fig. + . The hole current shape simulation result for MIP 
$i(t)=i_{e}(t)+i_{p}(t)$

Fig. 5. shows the total induced current by MIIP in a pt$n-n+$ silicon detector.

Comparison between the MIP current shape calculated by the analytical solutions in this paper and that by the semi-analytical method in Ref. [1] is shown in Fig. 6. It is clear that current shapes are similar and the difference is caused by the fiact that in Ref. [1], carricr salturation velocities in the high field was considered, resulting in longer charge collection time.

SIMULATED CURRENT SHAPES WITH TRAPPING $d=300 \mathrm{um}, A=1.0 \mathrm{~cm} 2, N d=1 E 12 / \mathrm{cm} 3$

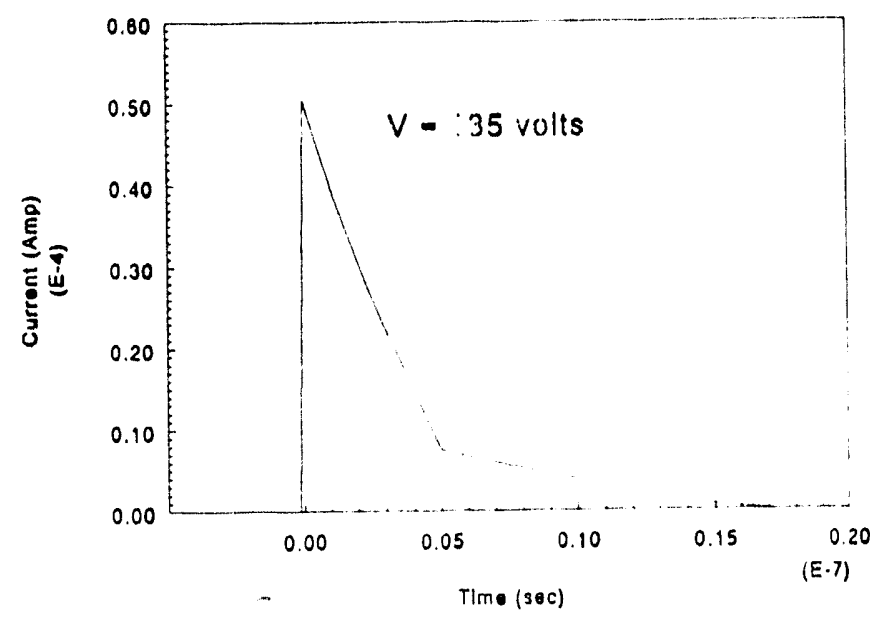

Fig. 5. The total current shape of simulation result for MIIP

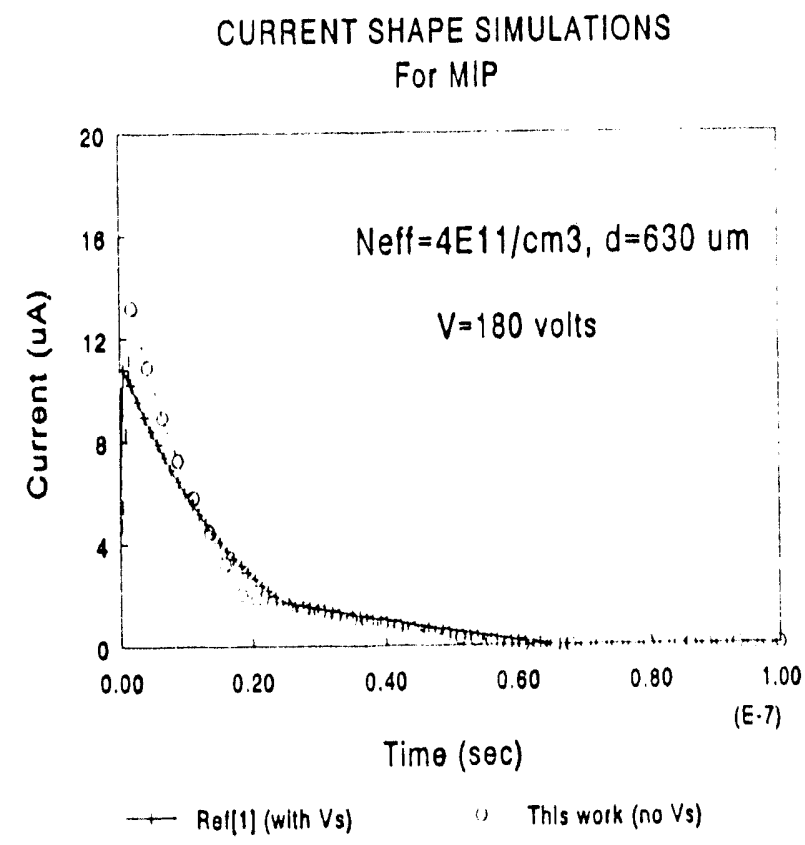

Fig. 6 Comparison between the antalytical solution of this study and the semi-analytical one of Ref.[1].

\section{Calculation of charge shape}

By integrating equation (20), one can get the induced charge shape by MIIP for the detector:

$$
Q(t)=\int_{0}^{1} i\left(t^{\prime}\right) d t^{\prime}
$$

$Q(t)$ can be calculated numerically and the computation result is shown in Fig. 7.

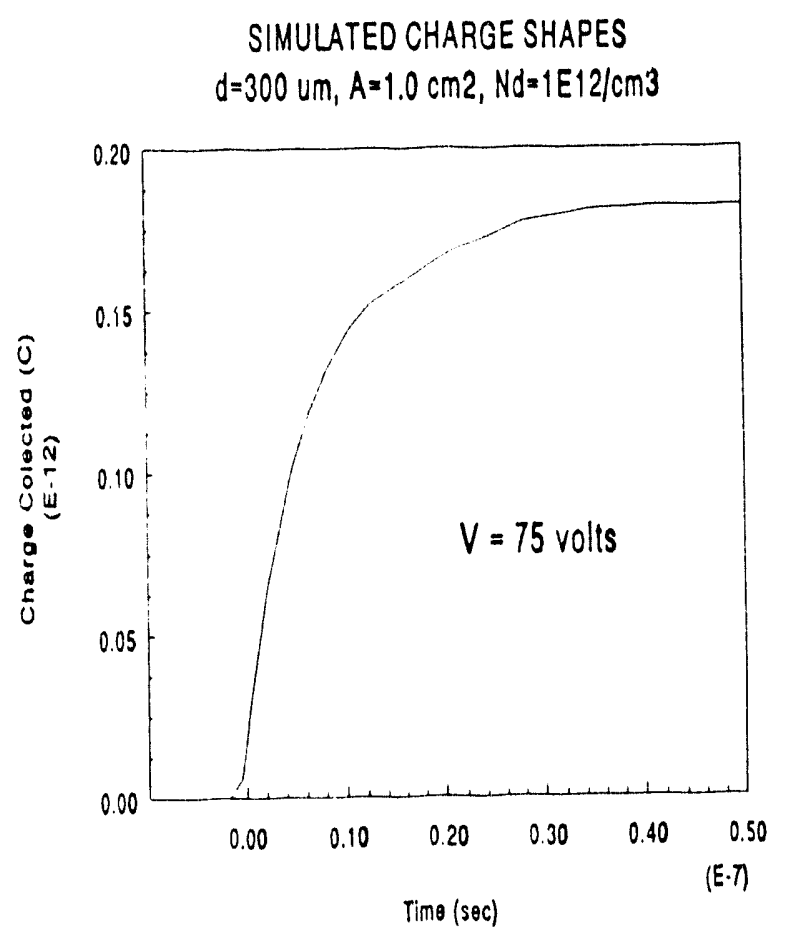

Fig. 7. The totil charge simulation result for MIP

\section{TRAPPING EFFECTS}

Decp levels in silicon detectors trap free carriers, which cause charge collection loss. This charge trapping effect is worse for detectors exposed to high fluence $\left(D_{n}>10^{13} \mathrm{n} / \mathrm{cm}^{2}\right)$ of neutron radiation that induce high concentrations of deep levels[3]. The trapping of a deep with an chergy level $E_{t}$ in the band gap is characterized by a trapping time constant $\tau_{l}[1]$ and eq. (6) can be modified as the following: 
$\frac{d n(x, t)}{d t}=\frac{\partial n}{\partial t}+\frac{\partial n}{\partial_{t} x} \frac{\partial x}{\partial t}-\frac{n}{\tau_{t}}$

This leads to:

$\frac{d n}{d t}=\frac{\mu \omega \rho}{\varepsilon \varepsilon_{0}}-\frac{n}{\tau_{t}}$

and

$n(t)=n_{0} e^{\frac{\mu \rho}{\varepsilon \varepsilon_{0}} t} e^{-\frac{t}{\tau_{t}}}$

Eqs. (11) and (17) can be rewritten as

$$
\begin{aligned}
& i_{e}^{(t)}=\frac{1}{d} \mu_{e} e n_{0} e^{\frac{\mu_{0} \rho}{\varepsilon \varepsilon_{0}}}, e^{-\frac{t}{\tau_{l}^{e}}} * \\
& *\left[E_{o}(d-\xi(t))-\frac{\rho}{2 \varepsilon \varepsilon_{0}}\left(d^{2}-\xi^{2}(t)\right)\right]
\end{aligned}
$$

$$
\begin{aligned}
& i_{p}^{(t)}=\frac{\mu_{p} p_{0}}{d} e^{-\frac{\left.\mu_{\mu}\right)}{\varepsilon \varepsilon_{0}}}, e^{-\frac{1}{t^{p}}} * \\
& *\left[E_{0} \xi_{p}(t)-\frac{\rho}{2 \varepsilon \varepsilon_{0}} \xi^{2}(t)\right]
\end{aligned}
$$

where $\tau_{t}^{e}$ and $\tau_{t}^{p}$ are trapping constants for clectrons and holes, respectively; and $\xi(t)$ and $\xi_{p}(t)$ are still defined by eqs. (13) and (18), respectively.

Fig. 8 shows the trapping effect on the current shipes for MIP. Obviously, more trapping (smaller $\tau_{t}$ ) means less area under the current curve and therefore less charge collection. This point is illustrated clearly in Fig. ". where charge shapes are plotted which various trapping time constants $\tau_{t}$ It has been known that $\tau_{t}$ is proportional inversely to the neutron fluencel $[1,3]$, which laids to smaller $\tau_{t}$ at higher neutron nuence and lherefore less charge collection.

\section{SIMULATED CURRENT SHAPES WITH TRAPPING \\ $d=300 u m, A=1.0 \mathrm{~cm} 2, N d=1 E 12 / \mathrm{cm} 3$}

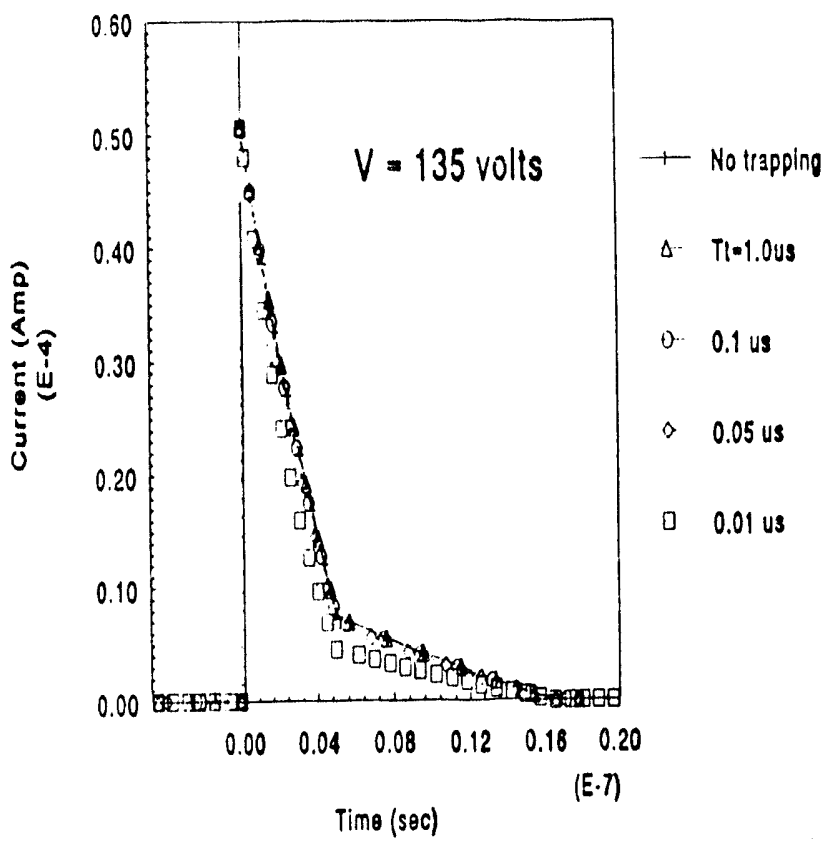

Fig. 8 Elfect of deep level trapping on the MIP current shapes

\section{SIMULATED CHARGE SHAPES WITH TRAPPING $d=300$ um, $A=1.0 \mathrm{~cm} 2, \mathrm{Nd}=1 \mathrm{E} 12 / \mathrm{cm} 3$}

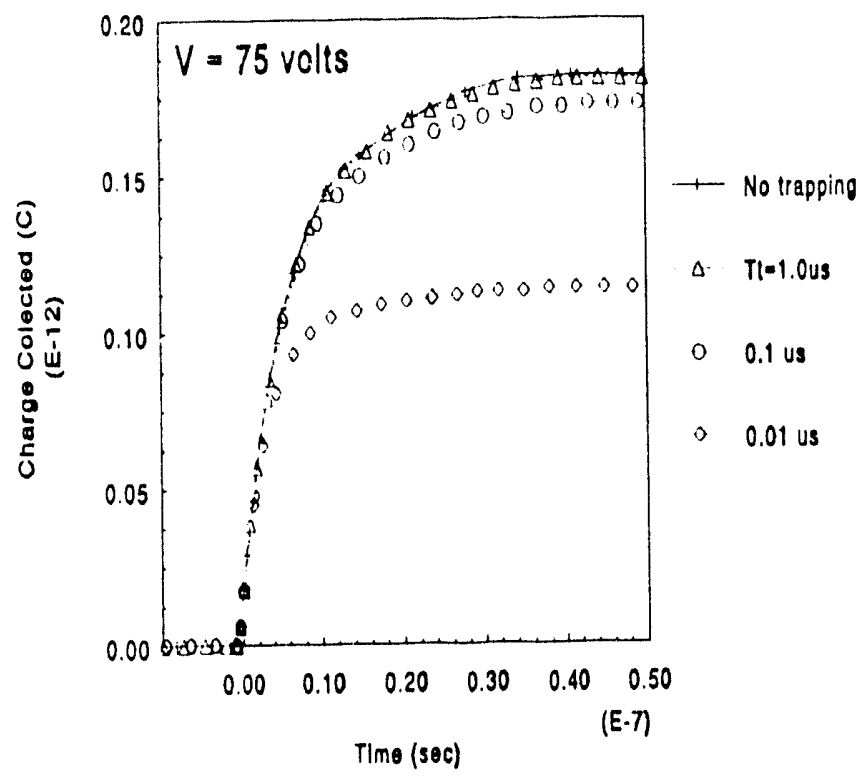

Fig. 9 Charge shapes at a given bias with various trapping constants 
For a detector irradiated to a certian neutron huence with given $\tau_{l}$ and doping concentration $\left(N_{d}\right.$ or $\left.N_{e, f f}\right)$. higher operating voltages would give shorter charge collection times and less trapping. This voltage or field effect is shown in Fig. 10. The full depletion vollige for this detector is ibout 68 volts and $\tau_{t}$ is set at $(1.01 \mu$ s. The charge collection at $\mathrm{V}=70$ volts is about $60 \%$, while it is $71 \%$ at 100 volts and $80 \%$ at 1.50 volts.

\section{SIMULATED CHARGE SHAPES WITH TRAPPING $d=300 \mathrm{um}, A=1.0 \mathrm{~cm} 2, N d=1 E 12 / \mathrm{cm} 3$}

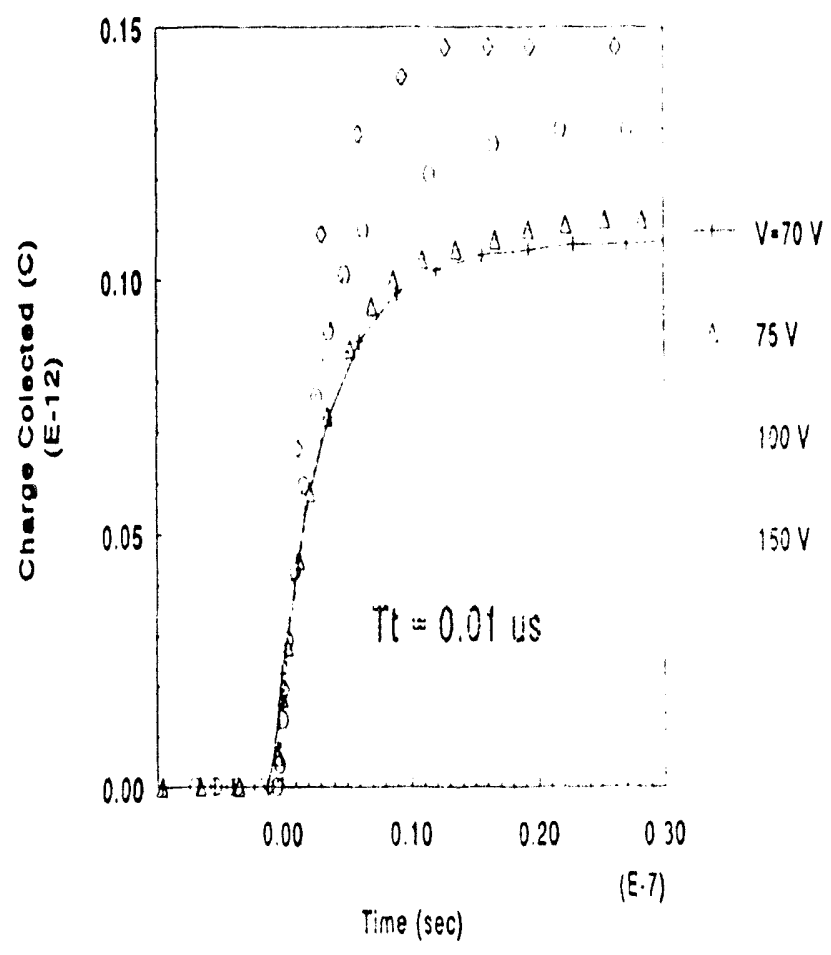

Fig. 10 Charge shapes for the same trapping time constimt but various biases $\left(V>V_{d}\right)$

As it is shown in Fig. 11, the charge collection of the detector has little dependence on the effective doping concentration $\left(N_{e f f}\right)$ in the space charge region(SCR). In other words, as long as the detector is biased well over the full depletion voltage, the field distribution in the SCR his little effect on the charge collection efficiency. This is a very important point given the fact that $N_{\text {eff }}$ hals a strong dependence on the neutron fluencelll.

\section{SIMULATED CHARGE SHAPES WITH TRAPPING $d=300$ um, $A=1.0 \mathrm{~cm} 2$}

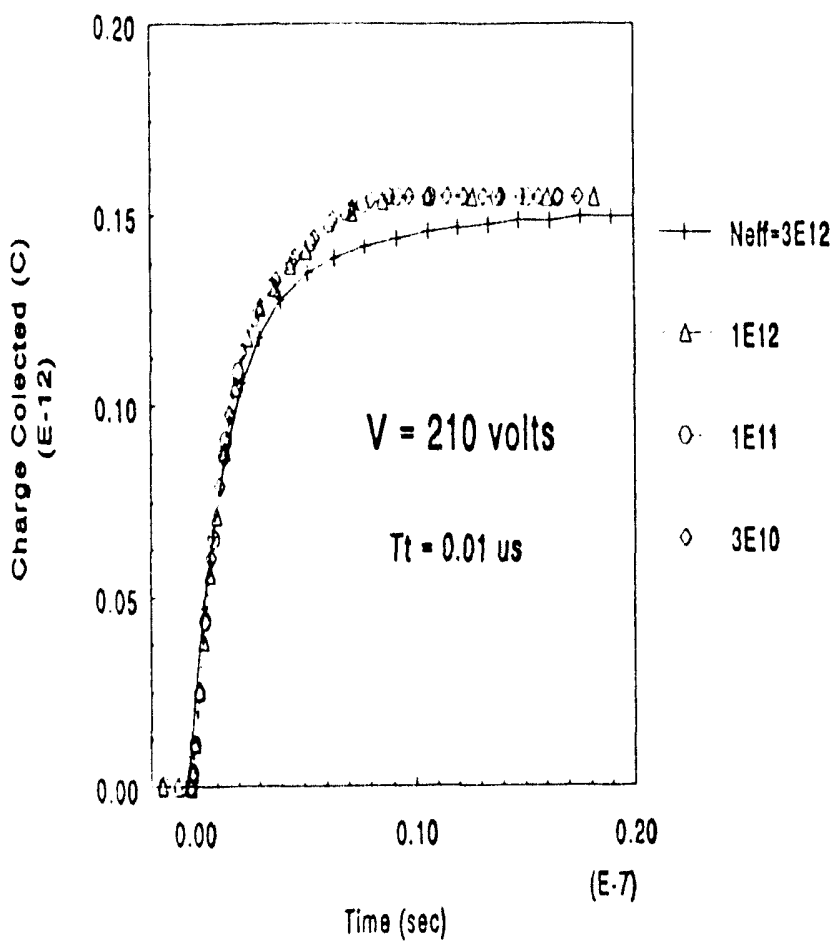

Fig. II Charge shape dependence on the effective doping concentration $\left(N_{\text {efff }}\right)$ in the space charge region.

\section{SUMMARY}

It has been shown that, for MIP, one can get analytical solutions for the electron and hole current shapes in a onedimensional problem. The results in this work are similar 10 those in reference 111, where a semi-analytical method was used. The trapping offects by deep levels introduced by displacement radiation has also been considered, and it does not affect annalytical nature of the solutions. The effect of carrier saturation velocity in the high electrical field can also be incorporated in the calculations, but one may only get numerical solutions.

\section{REFERENCES}

11) Z. Li and H. W. Kraner, "Modeling and Simulation of Charge Collection Properties for Neutron Irradiated Silicon Detectors", BNL-48067, paper presented at the Third International Conference on Advanced Technology and Particle Physics, Como, Italy, June 22 26, 1992. Nucl. Phys. B, 32(1993), p398. 
(2) S. M. Sze, "Plysics of Semiconductor Derices", 2nd Edition, John Willey lnc, NY (1981).

(3) H. W. Kraner, Z. Li, and E. Fretwurst, "The use of the Signal Current Pulse Shape to Study the Internial Electrical Field Profile and Trapping Effects in Neutron Diamiged Silicon Detectors," BNL-47506, Nucl. Instr. and Meth., A 326, 350(1993). 

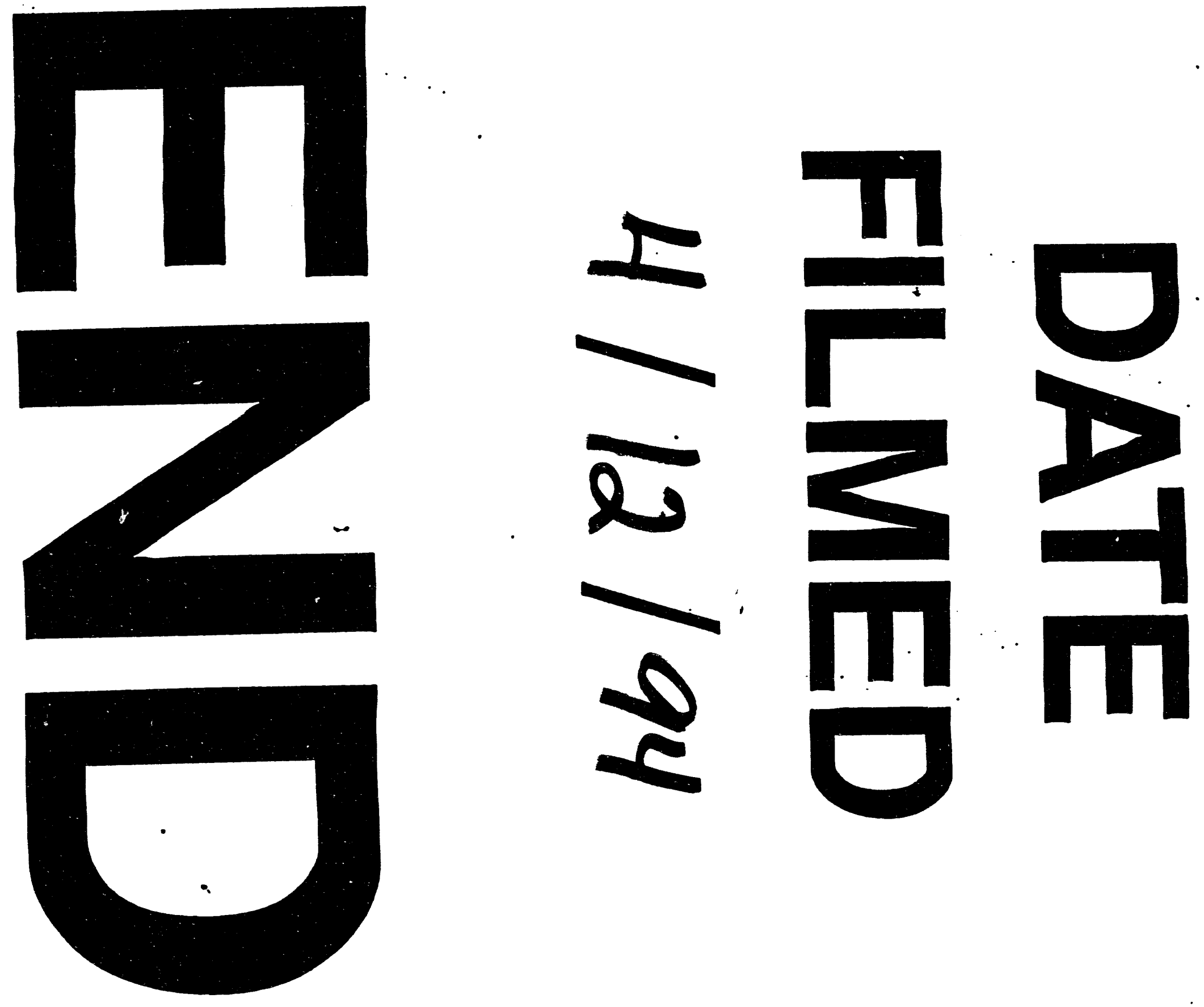


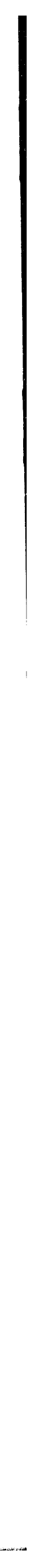

